\title{
Full command of a network by a new node: some results and examples
}

\author{
Clara Grácio ${ }^{1 *}$ (D) Sara Fernandes ${ }^{1}$ and Luís Mário Lopes²
}

\author{
${ }^{*}$ Correspondence: \\ mgracio@uevora.pt \\ 1 Department \\ of Mathematics, Universidade \\ de Évora and CIMA-UE \\ Rua Romão Ramalho, 59, \\ 7000-671 Évora, Portugal \\ Full list of author information \\ is available at the end of the \\ article
}

\begin{abstract}
We consider that a network of chaotic identical dynamical systems is connected to a new node. Depending on some properties of the network and on the way that connection is made, the new node may control the network. We consider a full-command connection and analyze the possibility of the network being full-commandable by the new node. For full-commandable networks, we define the full-command-window, a set that includes some of the values that the coupling strength of the new node may assume. We present several results and examples that enlight us how a network can become more vulnerable or resistant to full-command.
\end{abstract}

Keywords: Chaotic dynamical systems, Networks, Complete synchronization, Control, Full-command-windows

\section{Introduction}

Networks of chaotic dynamical systems are present in a wide range of situations, so that the study of their behavior is a very important matter. The synchronization is a relevant feature of that behavior [1-5]. Moreover, a relevant feature of chaotic dynamical system is its vulnerability or resistance to be controlled, with several ways that the control may be done [6-13]. So, it is also relevant to study the possibility that network synchronizes in a controlled way. In a previous paper [14], we considered that the control is made by adding a new node. In fact, if the new node is connected in an appropriate way to the network and if the network has some special properties, the new node can control the network. We considered that the new node is connected in what we called a full-commanded way. In "Full-commanding a network", we recall the results presented in that paper, while in "Examples of full-commanding and another result" we illustrate them by their application to some particular situations that provide us more insight into how a network is more vulnerable or resistant to a full-command, suggesting us a new result.

\section{Full-commanding a network}

There are many situations in which identical systems are connected in a linear way. We consider such a network, i.e., a network of the following type: 


$$
\begin{aligned}
x_{i}(t+1) & =f\left(x_{i}(t)\right)+\sum_{\substack{j=1 \\
j \neq i}}^{n} a_{i j} \cdot\left[f\left(x_{j}(t)\right)-f\left(x_{i}(t)\right)\right], \quad \forall_{i=1, \ldots, n} \\
& \Leftrightarrow \\
x_{i}(t+1) & =\left(1-\sum_{\substack{j=1 \\
j \neq i}}^{n} a_{i j}\right) \cdot f\left(x_{i}(t)\right)+\sum_{\substack{j=1 \\
j \neq i}}^{n} a_{i j} \cdot f\left(x_{j}(t)\right), \quad \forall_{i=1, \ldots, n} \\
& \Leftrightarrow \quad \vec{f}(\vec{x}(t)),
\end{aligned}
$$

where $n$ is the number of identical systems connected, $f$ is their free dynamic, $\vec{x}(t)=\left[\begin{array}{llll}x_{1}(t) & x_{2}(t) \ldots x_{n}(t)\end{array}\right]^{T}, \quad \vec{f}(\vec{x}(t))=\left[\begin{array}{l}f\left(x_{1}(t)\right) f\left(x_{2}(t)\right) \ldots f\left(x_{n}(t)\right)\end{array}\right]^{T}$, $a_{i i}=-\sum_{\substack{j=1 \\ j \neq i}}^{n} a_{i j}$ and $A=\left[a_{i j}\right]$. Such a network admits the completely synchronized solution $s(t) \cdot \overrightarrow{1}$, with $\overrightarrow{1}=[11 \ldots 1]^{T}$ and $s(t)$ such that $s(t+1)=f(s(t))$. In fact, since $\lambda=0$ is an eigenvalue of the coupling matrix $A$, corresponding to the eigenvector $\overrightarrow{1}$, we have

$$
\begin{aligned}
\left(I_{n}+A\right) \cdot f(s(t) \cdot \overrightarrow{1}) & =\left(I_{n}+A\right) \cdot f(s(t)) \cdot \overrightarrow{1} \\
& =s(t+1) \cdot(\overrightarrow{1}+A \cdot \overrightarrow{1})=s(t+1) \cdot \overrightarrow{1}
\end{aligned}
$$

Adding a new identical dynamical system, $y$, to the network, we want to analyze the possibility that, after a transient period, this new node imposes its iterates to all the others. In a coupling, we obtain that using a one-way connection with a coupling strength greater than $1-e^{-h}$, where $h$ is the Lyapunov exponent of the coupled systems [17]. So, we consider that the new node is one-way connected to all nodes of the network, i.e., we consider the new network

$$
\begin{aligned}
& \left\{\begin{array}{l}
x_{i}(t+1)=f\left(x_{i}(t)\right)+\sum_{\substack{j=1 \\
j \neq i}}^{n} a_{i j} \cdot\left[f\left(x_{j}(t)\right)-f\left(x_{i}(t)\right)\right]+\epsilon \cdot\left[f\left(y(t)-f\left(x_{i}(t)\right)\right], \quad \forall_{i=1, \ldots, n}\right. \\
y(t+1)=f(y(t))
\end{array}\right. \\
& \left\{\begin{array}{l}
x_{i}(t+1)=\left(1-\sum_{\substack{j=1 \\
j \neq i}}^{n} a_{i j}-\epsilon\right) \cdot f\left(x_{i}(t)\right)+\sum_{\substack{j=1 \\
j \neq i}}^{n} a_{i j} \cdot f\left(x_{j}(t)\right)+\epsilon \cdot f(y(t)), \quad \forall_{i=1, \ldots, n} \\
y(t+1)=f(y(t))
\end{array} \Leftrightarrow\right. \\
& \begin{array}{c}
\Leftrightarrow \\
\overrightarrow{x_{0}}(t+1)=\left(I_{n+1}+A_{0}\right) \cdot \vec{f}\left(\overrightarrow{x_{0}}(t)\right),
\end{array}
\end{aligned}
$$

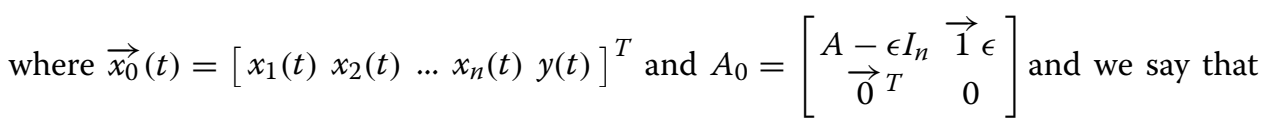
the new node $y$ is full-commanding the network $A$. 
In order that the new node imposes its free evolution to all the others nodes, it is needed that $y(t) \cdot \overrightarrow{1}$, with $y(t)$ such that $y(t+1)=f(y(t))$, be an exponentially stable solution of (2). The following proposition determines conditions for that to happen.

Proposition 1 If $\left|\epsilon-\left(1+\lambda_{i}\right)\right|<e^{-h}$, for all the eigenvalues $\lambda_{i}$ of the diagonalizable coupling matrix $A$ of the network (1), then the network is full-commanded by a new node $y$, i.e., $\overrightarrow{x_{0}}(t)=y(t) \cdot \overrightarrow{1}$, with $y(t)$ such that $y(t+1)=f(y(t))$, is an exponentially stable solution of (2). If $\left|\epsilon-\left(1+\lambda_{i}\right)\right|>e^{-h}$ for an eigenvalue $\lambda_{i}$ then network (1) is not fullcommanded by a new node.

As proved in [14] this is just the result of applying the following proposition (a variation of similar others $[15,16])$ to network (2), i.e., the result of considering that the matrix $A$ of the following proposition is $A_{0}$.

Proposition 2 Considering the dynamical network (1) with $A$ a diagonalizable matrix such that $\lambda_{1}=0$ is an eigenvalue of multiplicity 1 , if all the other eigenvalues $\lambda_{i}(i=2, \ldots, n)$ are such that $\left|1+\lambda_{i}\right|<e^{-h}$, where $h$ is the Lyapunov exponent of the nodes, then the completely synchronized solution $\vec{x}(t)=x_{1}(t) \cdot \overrightarrow{1}$, with $x_{1}(t)$ satisfying $x_{1}(t+1)=f\left(x_{1}(t)\right)$, is exponentially stable. If $\left|1+\lambda_{i}\right|>e^{-h}$ for a non-zero eigenvalue $\lambda_{i}$ then there is no exponentially stable completely synchronized solution.

All the same, we define full-command-window and full-commandable network, in the following way.

Definition 1 We define full-command-window $(F C W)$ of the network (1) as the open set of values of the commanding coupling strength $\epsilon \in[0,1]$ for which the synchronized solution $\overrightarrow{x_{0}}(t)=y(t) \cdot \overrightarrow{1}$ is an exponentially stable solution of $(1)$. If $F C W \neq \varnothing$, we say that network (1) is full-commandable.

Proposition 1 determines that

$$
F C W=\bigcap_{i=1}^{n}\left\{\epsilon \in[0,1]:\left|\epsilon-\left(1+\lambda_{i}\right)\right|<e^{-h}\right\} .
$$

As we noted in [14], there are networks that are not full-commandable, for instance the ones that have a diagonalizable coupling matrix $A$ with an eigenvalue such that $\operatorname{Im}(\lambda)>e^{-\mu}$ or such that its distance to another eigenvalue is greater than $2 e^{-\mu}$. We also presented in that paper the following results that are useful for obtaining the ones we add in this paper.

Proposition 3 Considering a network (1) such that $A$ is diagonalizable, all its eigenvalues are real and $\lambda_{n}$ is the smallest one, then the network is full-commandable if $\lambda_{n}>-2 e^{-h}$ and FCW reduces to

$$
F C W=] 1-e^{-h}, 1+\lambda_{n}+e^{-h}[\cap[0,1] .
$$


Proposition 4 A completely disconnected network, i.e., a network (1) with a zero matrix $A$, is full-commandable and its full-command-window is $\left.] 1-e^{-h}, 1\right]$.

Proposition 5 A completely connected network, i.e., a network (1) with

$$
A=c \cdot\left[\begin{array}{ccccc}
-(n-1) & 1 & 1 & \ldots & 1 \\
1 & -(n-1) & 1 & \ldots & 1 \\
1 & 1 & -(n-1) & \ldots & 1 \\
\ldots & \ldots & \ldots & \ldots & \ldots \\
1 & 1 & 1 & \ldots & -(n-1)
\end{array}\right],
$$

where $c$ is the global coupling strength, is full-commandable if $n c<2 e^{-h}$ and its full-command-window is $] 1-e^{-h}, 1-n c+e^{-h}[\cap[0,1]$.

We note that for a network satisfying the conditions of any of the these propositions in order to be full-commandable, the smallest commanding coupling constant is $\epsilon=1-e^{-h}$, a value that does not depend on the structure of the network, it only depends on the dynamic of the nodes. This value is exactly the same that it is needed in a one-way linear coupling for a dynamical system to command the other one [17]. Further, a completely connected network is as more resistant to a full-command as greater is the network (i.e., as greater is $n$ ) and as stronger are the connections between the dynamical systems (i.e., as greater is $c$ ).

\section{Examples of full-commanding and another result}

Now we consider several examples of networks, not only to illustrate the previous results, but also to get insight into some aspects of the behavior of such a way of commanding a network. In doing so, there is a practical problem related to the fact that when dynamical systems with bounded iteration intervals are connected, some iterations may assume values outside the iteration interval. Instead of restricting the coupling strength $\epsilon$ of the new node so that does not happen, we adopt a more permissive strategy: we let that coupling strength assume any value in $[0,1]$ and when there are iterations that leave the iteration interval, we put them again in the iteration interval in a random way. This way we do not discard any exponentially stable full-commanded solutions due to the boundness of the iteration interval.

We consider networks of four logistic dynamical systems [18] and we start by analyzing the two referred extreme situations: a completely disconnected network and a completely connected one. For the completely connected network, we use three values of the coupling strength, namely $c=0.1, c=0.2$ and $c=0.27$. In Figs. 1 and 2, we sketch the network and present the graphs of the iterates $D(t)=\frac{1}{4} \cdot\left(\left|x_{1}(t)-y(t)\right|+\left|x_{2}(t)-y(t)\right|+\left|x_{3}(t)-y(t)\right|+\left|x_{4}(t)-y(t)\right|\right)$. If the network is fully commanded by the new node, then after a transient period $D(t) \simeq 0$, and that is why we just register the iterates after that period, namely for $t>100$. So, we are able to visualize the full-commanded windows that this numerical approach provides, i.e., the values of $\epsilon$ for which $D(t) \simeq 0$. As determined by Proposition 4, the completely disconnected network is full-commandable and the smallest coupling strength that is required to the new node is $\epsilon=0.5$. In fact, since the Lyapunov exponent of the logistic map is $h=\ln 2$, that value is $1-e^{-h}=0.5$. This is also its value for 


\section{4}

3

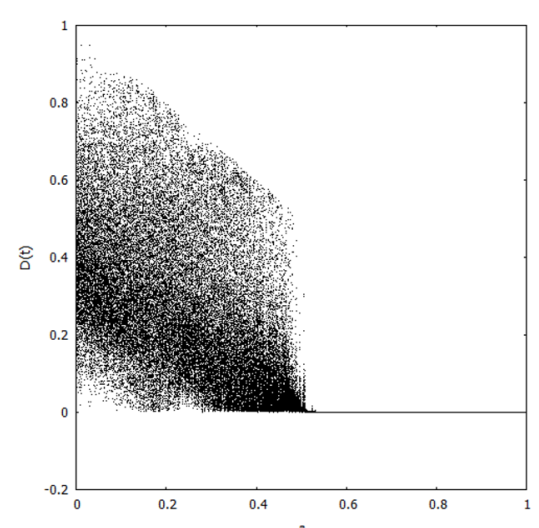

Fig. 1 Iterates $D(t)$ for a completely disconnected network as a function of $\epsilon$
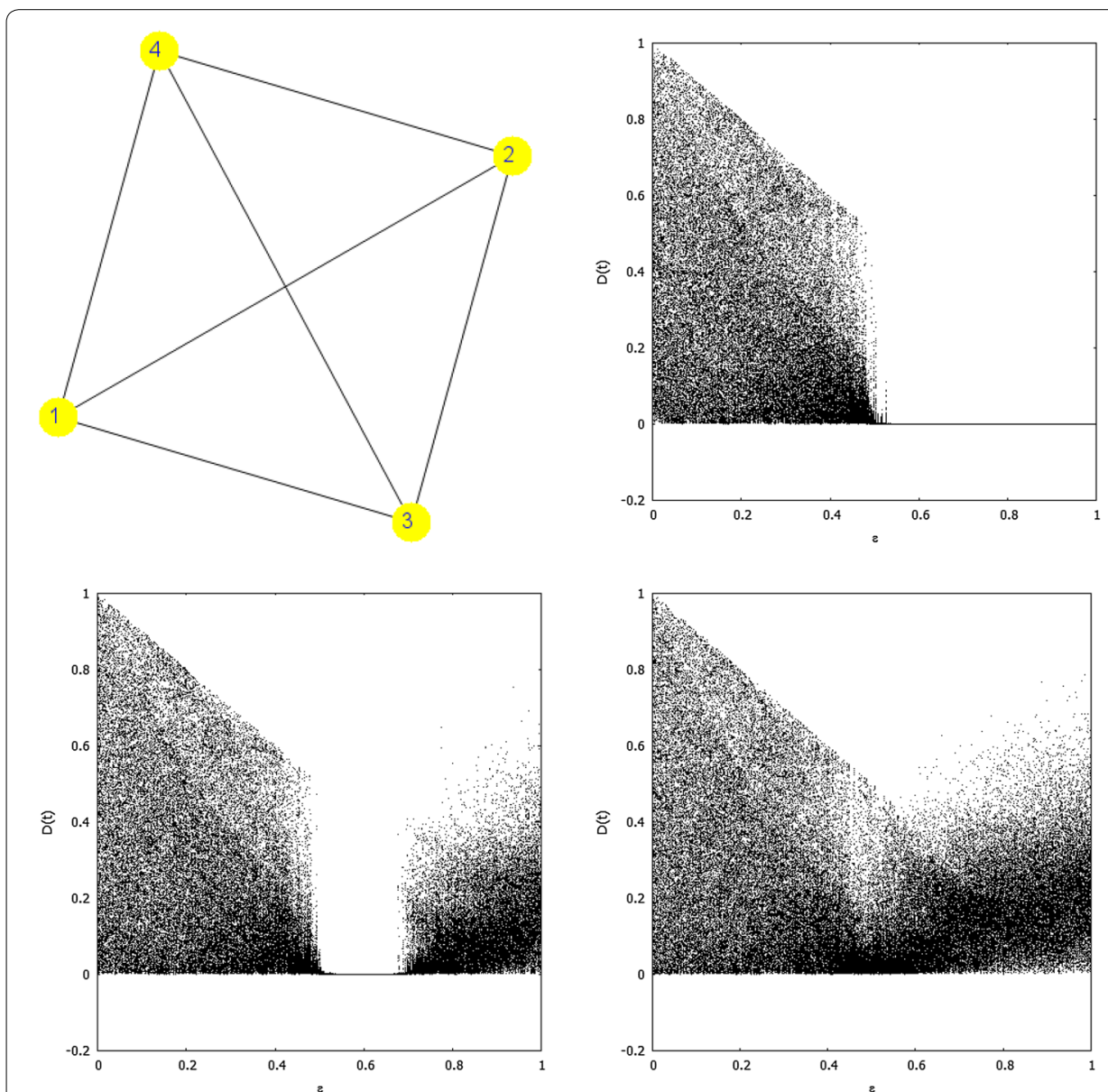

Fig. 2 Iterates $D(t)$ for a completely connected network as a function of $\epsilon$ for $c=0.1$ (top right), $c=0.2$ (bottom left) and $c=0.27$ (bottom right) 
the completely connected network, as Proposition 5 states, but this other network is only full-commandable if $c<\frac{2 e^{-h}}{n}$, i.e., if $c<0.25$. That is why, in Fig. 2, the full-command-window disappears for $c=0.27$. For $c=0.1$ and $c=0.2$, the full-commandwindows are ]0.5, 1] and ]0.5, 0.7[, respectively, as determined by Proposition 5.

Now, we consider two other networks with four nodes, neither as disconnected as a completely disconnected one nor as connected as a completely connected one, namely a network "in line" and a network "in star", corresponding to the sketches shown in Figs. 3 and 4 and, respectively, to the coupling matrixes $A_{L}=c \cdot\left[\begin{array}{cccc}-1 & 1 & 0 & 0 \\ 1 & -2 & 1 & 0 \\ 0 & 1 & -2 & 1 \\ 0 & 0 & 1 & -1\end{array}\right]$ and $A_{S}=c \cdot\left[\begin{array}{cccc}-3 & 1 & 1 & 1 \\ 1 & -1 & 0 & 0 \\ 1 & 0 & -1 & 0 \\ 1 & 0 & 0 & -1\end{array}\right]$ (used for instance in [16]). They have both three connections, but the way they are done determines a different resistance to a full-command. To highlight this, we considered for both of the networks the same three values of the coupling strength $c$ that we used for the completely connected network, namely $c=0.1$,

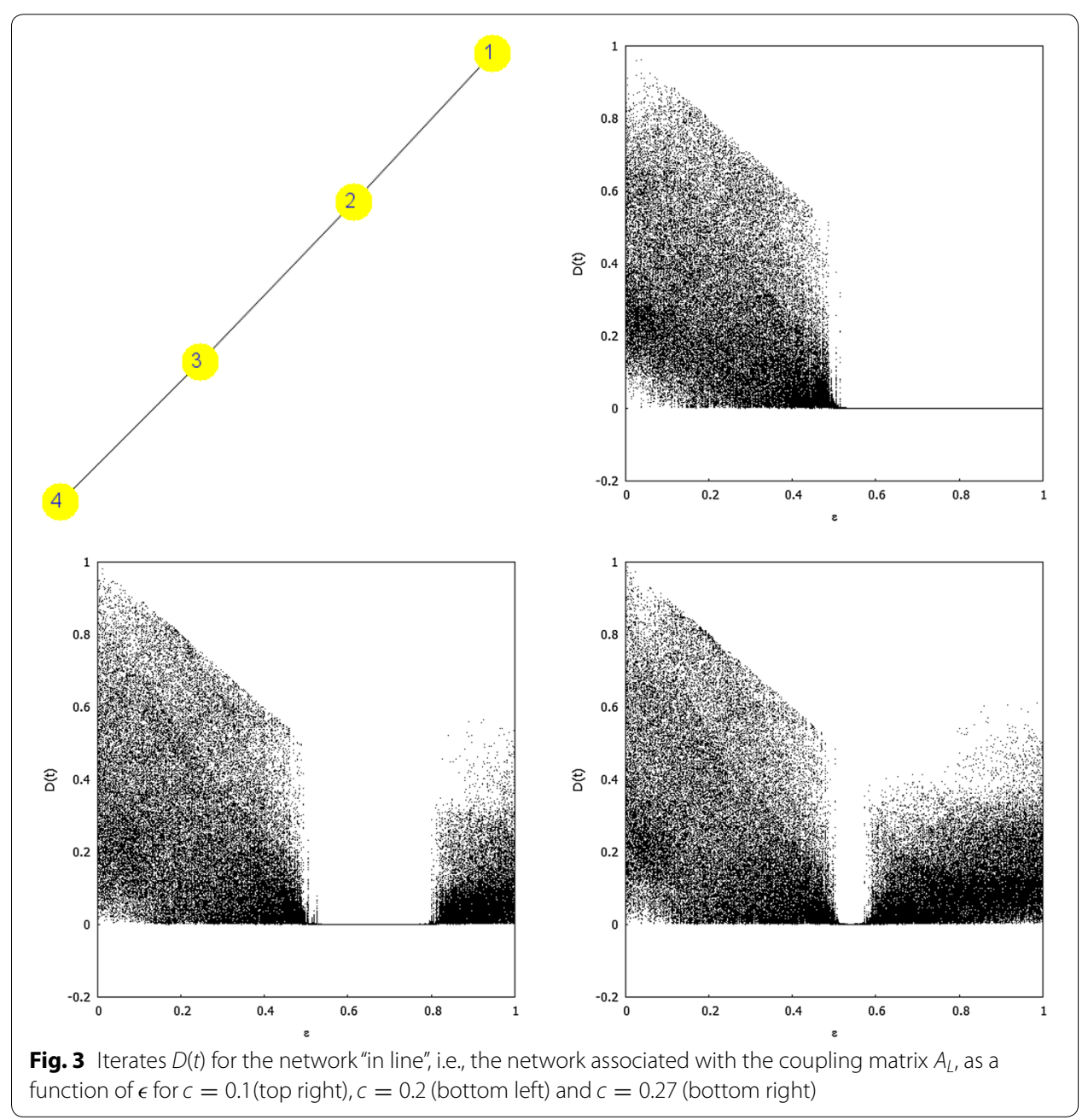




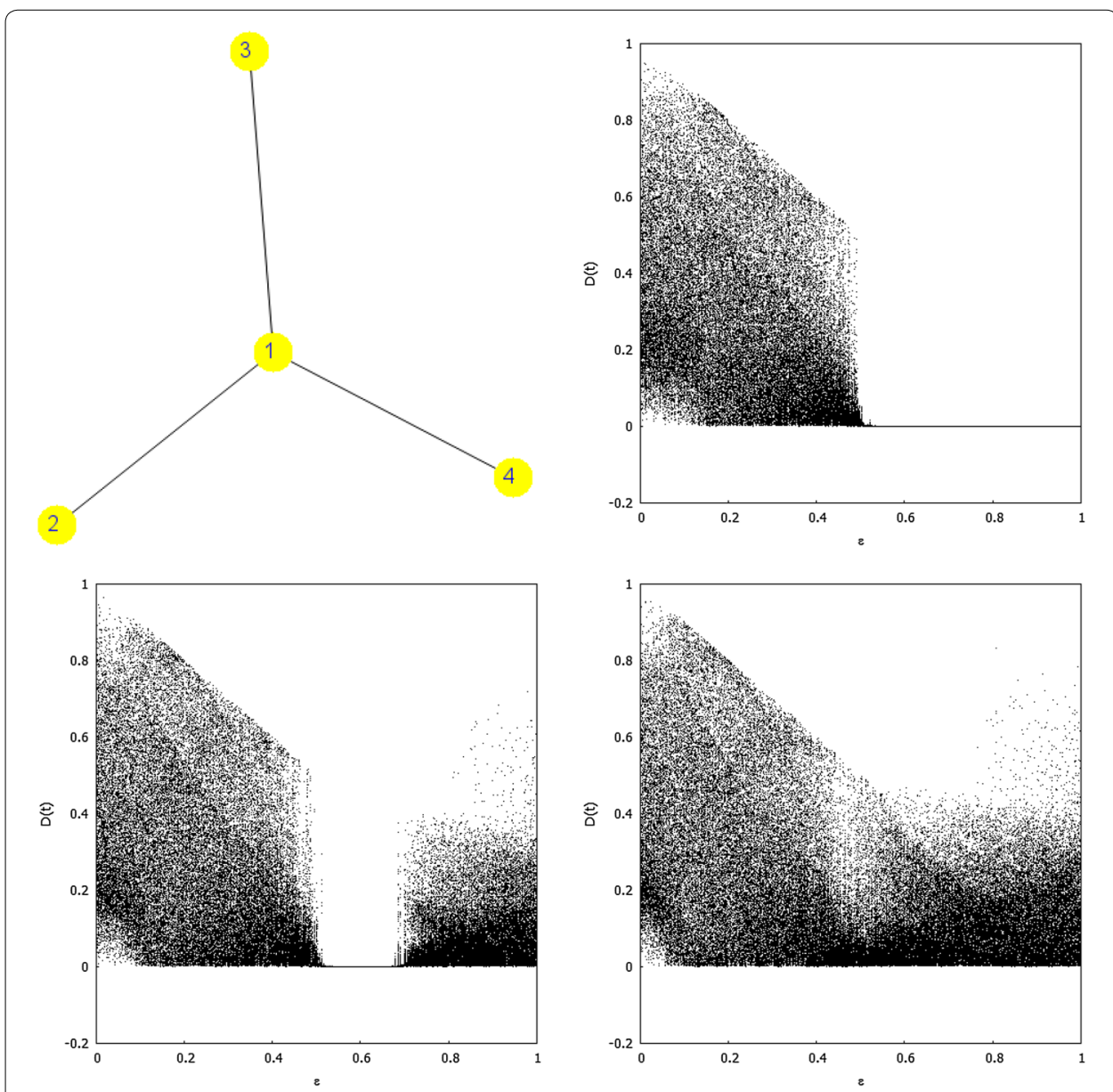

Fig. 4 Iterates $D(t)$ for the network "in star", i.e., the network associated with the coupling matrix $A_{S}$, as a function of $\epsilon$ for $c=0.1$ (top right), $c=0.2$ (bottom left) and $c=0.27$ (bottom right)

$c=0.2$ and $c=0.27$, and we show in Figs. 3 and 4 the values of iterates of $D(t)$ after the transient period.

Proposition 3 confirms the full-command-windows that the graphs exhibit. In fact, since the eigenvalues of $A_{1}$ are $\lambda_{1}=0, \lambda_{2}=(-2+\sqrt{2}) c, \lambda_{3}=-2 c, \lambda_{4}=(-2-\sqrt{2}) c$, the network "in line" is full-commandable for $c<\frac{1}{2+\sqrt{2}} \simeq 0.293$, so that for all the three values of $c$ we obtain a non-empty full-command-window with the already referred left endpoint 0.5 , while the right endpoints are $1,0.817$ and 0.578 , since the values of $1+\lambda_{4}+e^{-h}$ for $c=0.1, c=0.2$ and $c=0.27$ are $1.159,0.817$ and 0.578 , respectively. In the same way, since the eigenvalues of $A_{2}$ are $\lambda_{1}=0, \lambda_{2}=\lambda_{3}=-c, \lambda_{4}=-4 c$, the network "in star" is full-commandable for $c<0.25$, so that the full-command-window for $c=0.27$ is empty, while the ones corresponding to $c=0.1$ and $c=0.2$ have right endpoints 1 and 0.7 , since $1+\lambda_{4}+e^{-h}$ for $c=0.1$ and $c=0.2$ are 1.1 and 0.7 , respectively.

These examples show that for networks with the same number of nodes, the same value of the coupling strength and the same number of connections, the resistance to a full-command depends on the way those connections are made, namely a network "in star" is more resistant to a full command than a network "in line". Further, the 
similarity between the results of Figs. 2 and 4 suggests that a network connected "in star" seems to be as resistant to a full command as a completely connected network where there are many more connections. This is in fact true and results from the following proposition.

Proposition 6 A network "in star" i.e., a network that has a node connected to all the other nodes, corresponding to a coupling matrix $A_{S}=c \cdot\left[\begin{array}{ccccc}-(n-1) & 1 & 1 & \ldots & 1 \\ 1 & -1 & 0 & \ldots & 0 \\ 1 & 0 & -1 & \ldots & 0 \\ \ldots & \ldots & \ldots & \ldots & \ldots \\ 1 & 0 & & \ldots & -1\end{array}\right]$ has a full-commanded-window $F C W \subset] 1-e^{-h}, 1-n c+e^{-h}[\cap[0,1]$.

Proof $A_{S}$ has as eigenvalue $\lambda=-n c$, since $A_{S} \cdot\left[\begin{array}{c}-(n-1) \\ 1 \\ 1 \\ \cdots \\ 1\end{array}\right]=-n c\left[\begin{array}{c}-(n-1) \\ 1 \\ 1 \\ \cdots \\ 1\end{array}\right]$. So, Proposition 3 determines that $F C W \subset] 1-e^{-h}, 1-n c+e^{-h}[\cap[0,1]$.

\section{Conclusions}

A network of linearly connected identical chaotic dynamical systems may be fullcommanded by a new identical node if its coupling matrix satisfies some conditions. Namely, if the coupling matrix is a diagonalizable matrix with real eigenvalues the full-commandability is determined just by its smallest eigenvalue and by the Lyapunov exponent of the dynamical systems. In that case, the smallest value of the coupling strength $\epsilon$ that determines the full-command only depends on the Lyapunov exponent of the dynamical systems. While a completely disconnected network is always fullcommandable, a completely connected one is as more difficult to command, as larger is the network (larger $n$ ) and as stronger are the connections between nodes (larger $c$ ). Even if a network has the same number of connections between nodes with the same coupling strength, a network "in line" is more vulnerable to a full-command than a network "in star". In fact, a network "in star" is as resistant to a full-command as a completely connected one.

Acknowledgements

Research partially sponsored by national funds through the Fundação Nacional para a Ciência e Tecnologia, PortugalFCT, under the project UID/MAT/04674/2013 (CIMA).

Authors' contributions

All authors prepared and discussed the results and contributed to the final manuscript. All authors read and approved the final manuscript.

Funding

Not applicable.

Availability of data and materials

Not applicable.

Competing interests

The authors declare that they have no competing interests. 


\begin{abstract}
Author details
${ }^{1}$ Department of Mathematics, Universidade de Évora and CIMA-UE, Rua Romão Ramalho, 59, 7000-671 Évora, Portugal. ${ }^{2}$ Mathematics Unit, ADM, Instituto Superior de Engenharia de Lisboa, Rua Conselheiro Emídio Navarro, 1, 1949-014 Lisbon, Portugal.
\end{abstract}

Received: 21 May 2019 Accepted: 3 December 2019

Published online: 26 December 2019

\title{
References
}

1. Pikovsky A, Rosenblum M, Kurths J. Synchronization, a universal concept in nonlinear sciences. Cambridge: Cambridge University Press; 2001.

2. Hasler M, Maistrenko YL. An introduction to the synchronization of chaotic systems: coupled skew tent maps. IEEE Trans Circuits Syst I. 1997;44(10):856-66.

3. Sushchik MM Jr, Rulkov NF, Abarbanel HDI. Robustness and stability of synchronized chaos: an illustrative model. IEEE Trans Circuits Syst I. 1997;44(10):867-73.

4. Rangarajan G, Ding MZ. Stability of synchronized chaos in coupled dynamical systems. Phys Lett A. 2002;296:204-9.

5. Ding MZ, Yang WM. Stability of synchronous chaos and on-off intermittency in coupled map lattices. Phys Rev E. 1997;56(4):4009-16.

6. Ott E, Grebogi C, Yorke J. Controlling chaos. Phys Rev Lett. 1990:64(11):1196-9.

7. Pyragas K. Continuous control of chaos by self-controlled feedback. Phys Lett A. 1992;170(6):421-8.

8. Nijmeijer H, Berghuis H. On Lyapunov control of the Duffing equation. IEEE Trans Circuits Syst I. 1995;42(8):473-7.

9. Bernardo M. An adaptive approach to the control and synchronization of chaotic systems. Int I Bifurc Chaos. 1996;6(3):557-68.

10. Boccaletti S, Grebogi C, Lai Y, Mancini H, Maza D. The control of chaos: theory and applications. Phys Rep. 2000;329:103-97.

11. Ge S, Wang C. Adaptive control of uncertain Chua's circuits. IEEE Trans Circuits Syst I. 2000;47(9):1397-402.

12. Harb A, Zaher Ashraf A. Nonlinear control of permanent magnet stepper motors. Commun Nonlinear Sci Numer Simul. 2004;9(4):443-58.

13. Ahlborn A, Parlitz U. Laser stabilization with multiple-delay feedback control. Opt Lett. 2006;31(4):465-7.

14. Grácio C, Fernandes S, Lopes L. Full-commanding a network: the dictator. In: Proc. 7th int. conf. complex networks and their applications VII, 2019; vol. 1. https://doi.org/10.1007/978-3-030-05411-3_41.

15. Feng J, Jost J, Qian M. Networks: from biology to theory. Berlin: Springer; 2007.

16. Li X, Chen G. Synchronization and desynchronization of complex dynamical networks: an engineering viewpoint. IEEE Trans Circuits Syst I. 2003;50(11):1381-90.

17. Lopes L, Fernandes S, Grácio C. Complete synchronization and delayed synchronization in couplings. Nonlinear Dyn. 2014;79:1615-24.

18. Lopes L, Fernandes S, Grácio C. "Windows of Synchronization" and "Non-chaotic Windows". ESAIM Proc Surv. 2014;46:161-74.

\section{Publisher's Note}

Springer Nature remains neutral with regard to jurisdictional claims in published maps and institutional affiliations.

\section{Submit your manuscript to a SpringerOpen ${ }^{\circ}$ journal and benefit from:}

- Convenient online submission

- Rigorous peer review

- Open access: articles freely available online

High visibility within the field

- Retaining the copyright to your article

Submit your next manuscript at $>$ springeropen.com 